\title{
Oxygen-rich Bis(trinitroethyl esters): Suitable Oxidizers as Potential Ammonium Perchlorate Replacement
}

Cornelia C. Unger, Marcel Holler, Burkhard Krumm, ${ }^{*}$ and Thomas M. Klapötke*

Department of Chemistry, Ludwig-Maximilian University of Munich, Butenandtstr. 5-13(D), D-81377 Munich, Germany

\section{SUPPORTING INFORMATION}

Table of Contents

1. General Experimental Details

SII

2. NMR Data of MaBTNE (1)

S III-IV

3. NMR Data of NABTNE (2)

S V-VI

4. Vibrational Spectroscopy

S VII

5. Microscope Images

S VII

6.1 Crystallographic Data of Nitraminodiacetic Acid

S VIII

6.2 Crystallographic Data of NABTNE (2)

S IX

7. Plots of DSC Measurements of NABTNE (2)

S X

8. Theoretical Calculations

S X-XII

9. References

S XIII 


\section{General Experimental Details}

${ }^{1} \mathrm{H},{ }^{13} \mathrm{C}$ and ${ }^{14} \mathrm{~N}$ NMR spectra were recorded on a $400 \mathrm{MHz}$ (Bruker $400 \mathrm{TR}$ ) spectrometer at ambient temperature, whereby the chemical shifts were determined with respect to external standards, $\mathrm{Me}_{4} \mathrm{Si}\left({ }^{1} \mathrm{H} 399.8 \mathrm{MHz} ;{ }^{13} \mathrm{C} 100.5 \mathrm{MHz}\right)$ and $\mathrm{MeNO}_{2}\left({ }^{14} \mathrm{~N} 28.9 \mathrm{MHz}\right)$. IR and spectra were measured with a PerkinElmer Spectrum One FTIR equipped an ATR device. Furthermore, Raman spectra were recorded using a Bruker MultiRAM FT Raman spectrometer with ND:YAG laser with excitation up to $1000 \mathrm{~mW}$ at $1064 \mathrm{~nm}$ respectively at ambient temperature. For the Raman spectra additionally glass tubes were used. Elemental analyses were obtained on an Elementar vario EL or Elementar Vario micro cube. Simple melting und decomposition points were measured with an OZM Research DTA 552-Ex apparatus with a linear heating rate of $5^{\circ} \mathrm{C} \mathrm{min}-1$ in a temperature range of 15 to $400{ }^{\circ} \mathrm{C}$ and checked by a Büchi Melting Point B430 apparatus (not corrected). For the stability evaluation; DSC measurements were carried out on a DSC $2+$ from Mettler Toledo in sealed high pressure crucibles. Furthermore, a TGA/DSC 3+ was used for the TGA measurements, whereby aluminum oxide crucibles were used. The sensitivity data towards impact and friction were obtained using a BAM drophammer $^{1}$ and BAM friction tester ${ }^{2}$. The low temperature single-crystal X-ray diffraction studies were performed on an Oxford XCalibur3 diffractometer with a generator (voltage $50 \mathrm{kV}$, current $40 \mathrm{~mA}$ ) and a Kappa CCDarea detector operating with Mo- $K \alpha$ radiation $(\lambda=0.7107 \AA)$. For solving the structure direct methods were used (e.g. SIR97) $)^{3-4}$ and refined by full-matrix least-squares on $F^{2}$ (SHELXL) ${ }^{5-6}$ implemented in the WINGX software package. ${ }^{7}$ All non-hydrogen atoms were refined anisotropically and the DIAMOND plots are shown with thermal ellipsoids at the 50\% probability level. CCDC 2009758 (nitraminodiacetic acid) and CCDC 2009759 (NABTNE) contains the supplementary crystallographic data for this paper. These data can be obtained free of charge from The Cambridge Crystallographic Data Centre via www.ccdc.cam.ac.uk/data_request/cif. 


\section{NMR Data of MaBTNE}

The ${ }^{1} \mathrm{H}$ and ${ }^{13} \mathrm{C}$ NMR shifts of malonic acid bis(2,2,2-trinitroethyl ester) were measured in various solvents and are listed in Table S1. Since MaBTNE is not stable in acetone, no shifts are listed for this solvent. As an example, the spectra including the ${ }^{14} \mathrm{~N} \mathrm{NMR}$ spectra in $\mathrm{CD}_{3} \mathrm{CN}$ are depicted.

Table S1. MaBTNE in various deuterated solvents. Values in ppm.

\begin{tabular}{llllll}
\hline Spectra/Solvent & DMSO & $\mathrm{MeOD}$ & $\mathrm{CD}_{3} \mathrm{CN}$ & $\mathrm{CDCl}_{3}$ \\
\hline${ }^{1} \mathrm{H}$ & $\mathrm{CH}_{2}$ & 5.97 & 5.79 & 5.60 & 5.45 \\
& $\mathrm{CH}_{2}$ & 3.96 & 3.72 & 3.68 & 3.63 \\
${ }^{13} \mathrm{C}$ & $\mathrm{CO}$ & 163.6 & 164.6 & 164.3 & 162.4 \\
& $\mathrm{C}\left(\mathrm{NO}_{2}\right)_{3}$ & 124.3 & 125.24 & 124.5 & 122.7 \\
& $\mathrm{CH}_{2}$ & 61.1 & 62.5 & 62.0 & 61.5 \\
& $\mathrm{CH}_{2}$ & 39.4 & 48.8 & 40.6 & 39.8 \\
\hline
\end{tabular}<smiles>CC(C)(C)OCC(=O)OCC(=O)CC(=O)OCC(=O)OO</smiles>

${ }^{1} \mathrm{H}$, acetonitrile $\mathrm{D}_{3}$

8 尔

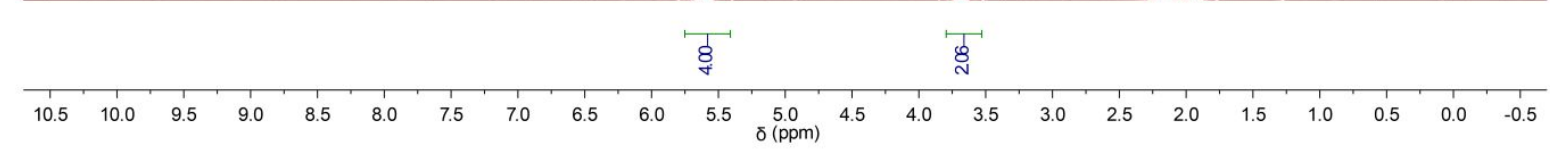




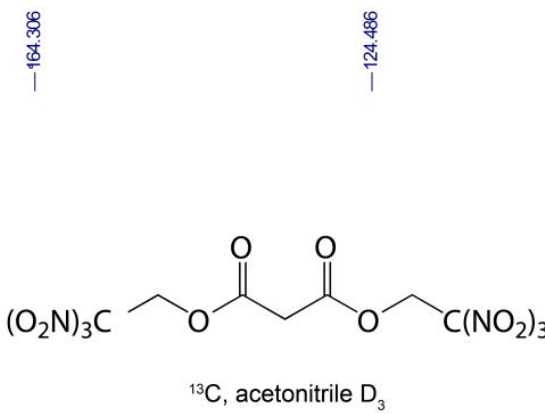

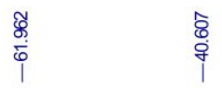

${ }^{13} \mathrm{C}$, acetonitrile $\mathrm{D}_{3}$
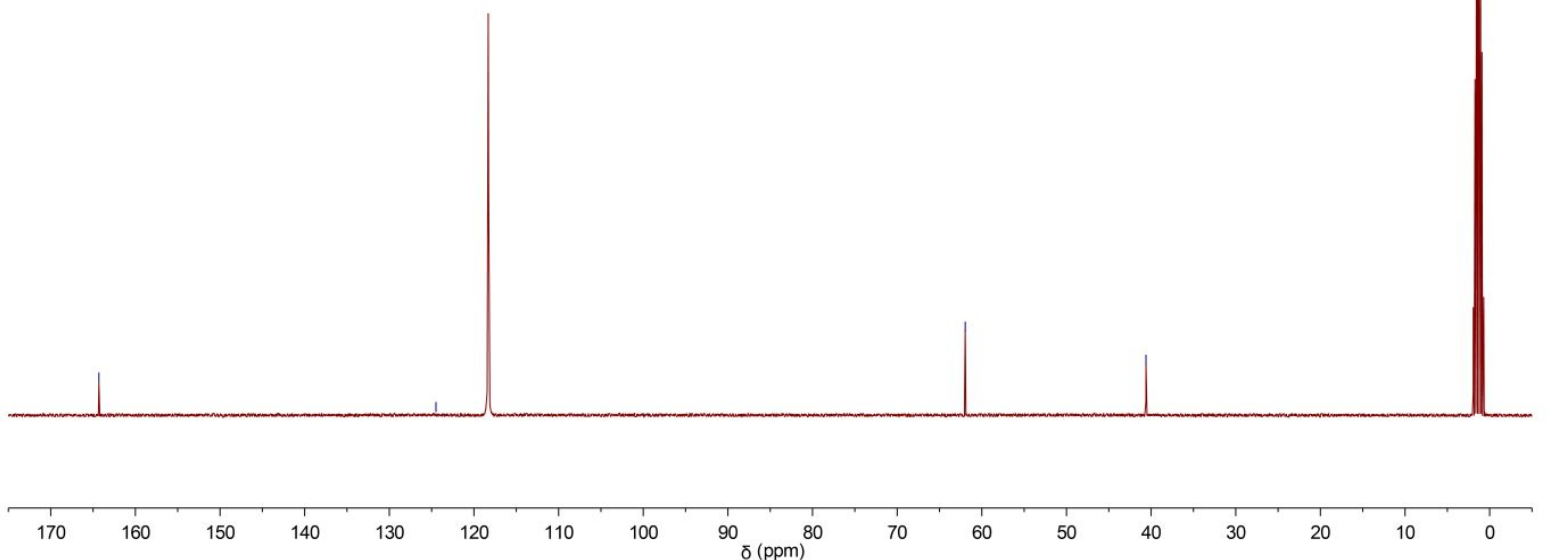

站

$\left(\mathrm{O}_{2} \mathrm{~N}\right)_{3} \mathrm{C} \frown \overbrace{\mathrm{O}} \overbrace{\mathrm{C}\left(\mathrm{NO}_{2}\right)_{3}}$

${ }^{14} \mathrm{~N}$, acetonitrile $\mathrm{D}_{3}$

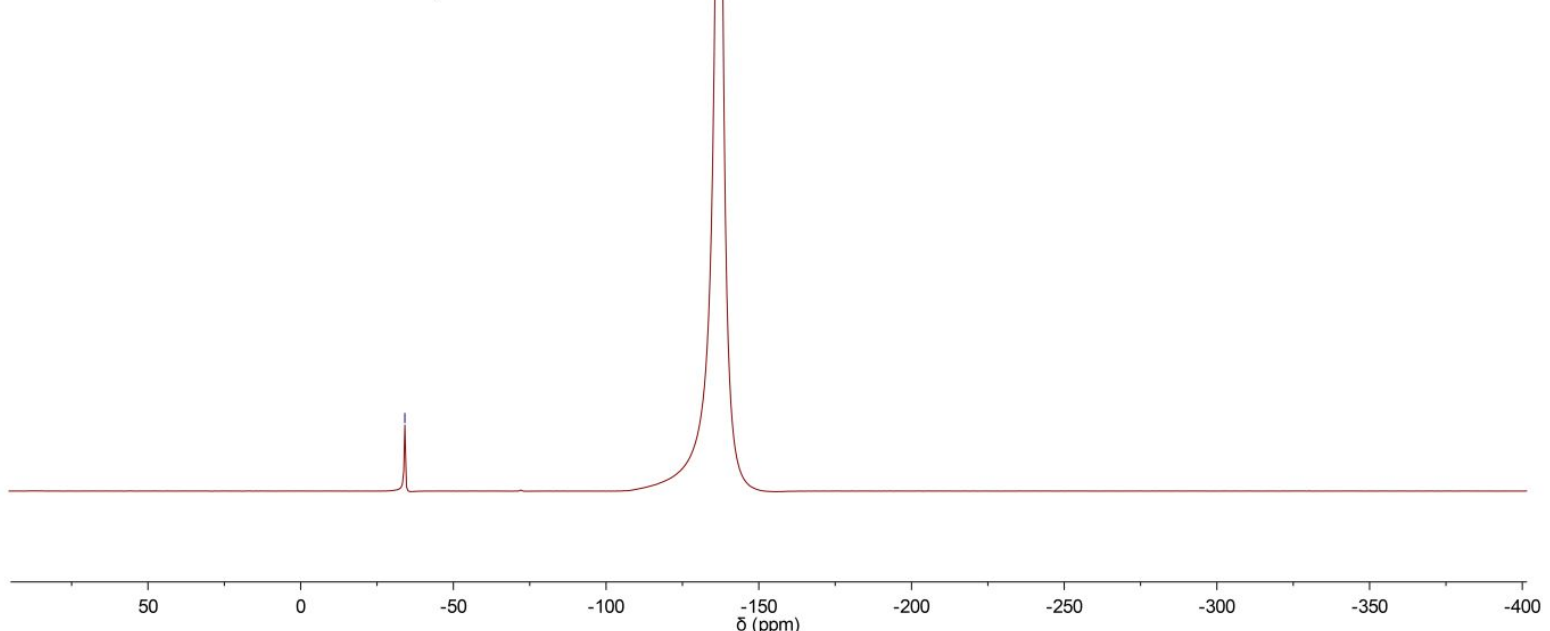




\section{NMR Data of NABTNE}

The ${ }^{1} \mathrm{H}$ and ${ }^{13} \mathrm{C}$ NMR shifts of nitramino diacetic acid bis(2,2,2-trinitroethyl ester) were measured in various solvents and are listed in Table S2. NABTNE is barely soluble in $\mathrm{CDCl}_{3}$, therefore only ${ }^{1} \mathrm{H}$ NMR resonances are listed. As an example, the spectra including the ${ }^{14} \mathrm{~N}$ $\mathrm{NMR}$ spectra in $\mathrm{CD}_{3} \mathrm{CN}$ are depicted.

Table S2. NABTNE in various deuterated solvents, shifts in ppm.

\begin{tabular}{lllllll}
\hline & & DMSO-D $_{6}$ & $\mathrm{MeOD}$ & $\mathrm{CD}_{3} \mathrm{CN}$ & $\mathrm{CDCl}_{3}$ & $\left(\mathrm{CD}_{3}\right)_{2} \mathrm{CO}$ \\
\hline${ }^{1} \mathrm{H}$ & $\mathrm{CH}_{2}$ & 6.03 & 5.84 & 5.64 & 5.50 & 5.96 \\
& $\mathrm{CH} H_{2}$ & 4.85 & 4.76 & 4.65 & 4.64 & 4.96 \\
${ }^{13} \mathrm{C}$ & $\mathrm{CO}$ & 164.7 & 166.1 & 165.6 & - & 165.7 \\
& $\mathrm{C}\left(\mathrm{NO}_{2}\right)_{3}$ & 124.3 & 129.1 & 124.7 & - & 131.8 \\
& $\mathrm{CH}_{2}$ & 61.2 & 62.6 & 62.1 & - & 62.3 \\
& $\mathrm{CH}_{2}$ & 52.6 & 53.6 & 53.7 & - & 53.5 \\
\hline
\end{tabular}

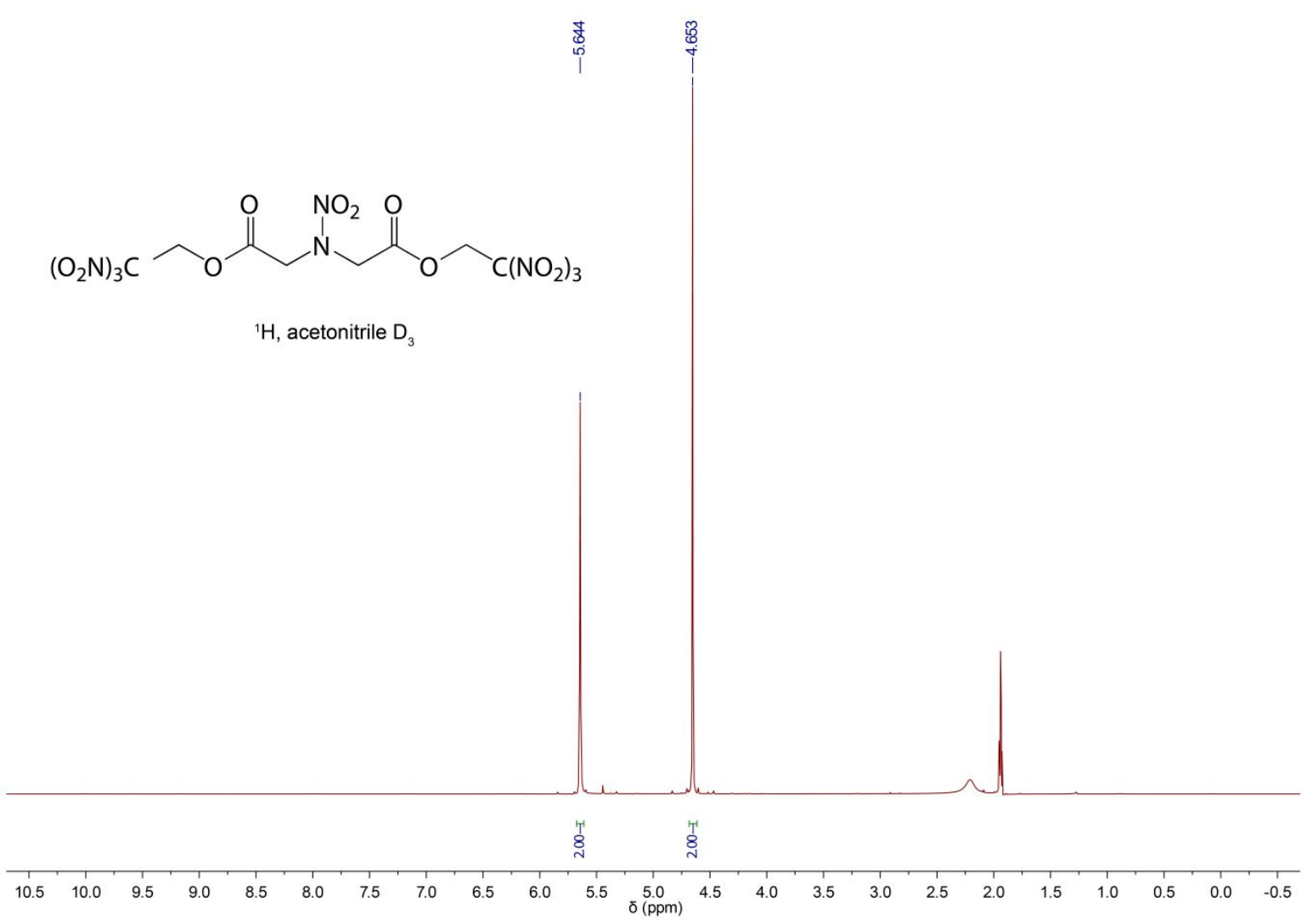



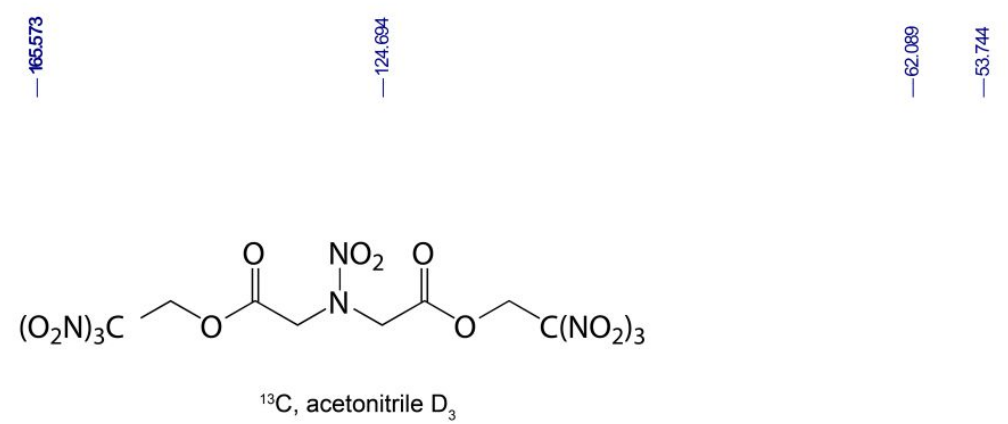

पิ

, acetonitrile D
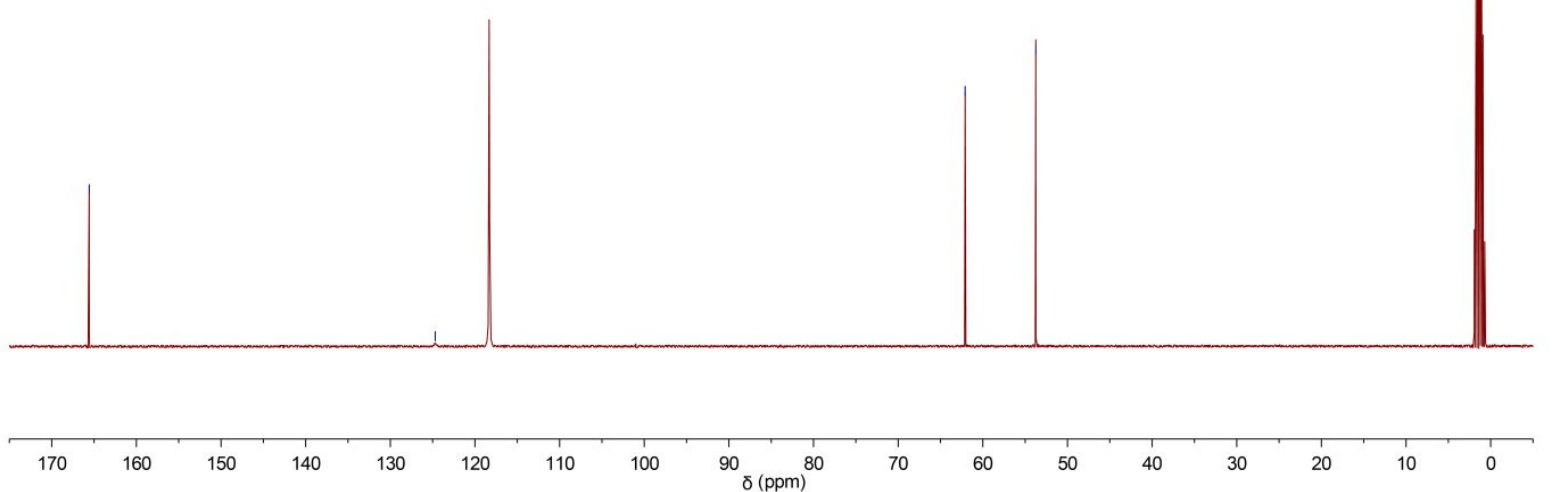

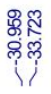

$\left(\mathrm{O}_{2} \mathrm{~N}\right)_{3} \mathrm{C} ح \mathrm{O}_{\mathrm{O}}$

${ }^{14} \mathrm{~N}$, acetonitrile $\mathrm{D}_{3}$

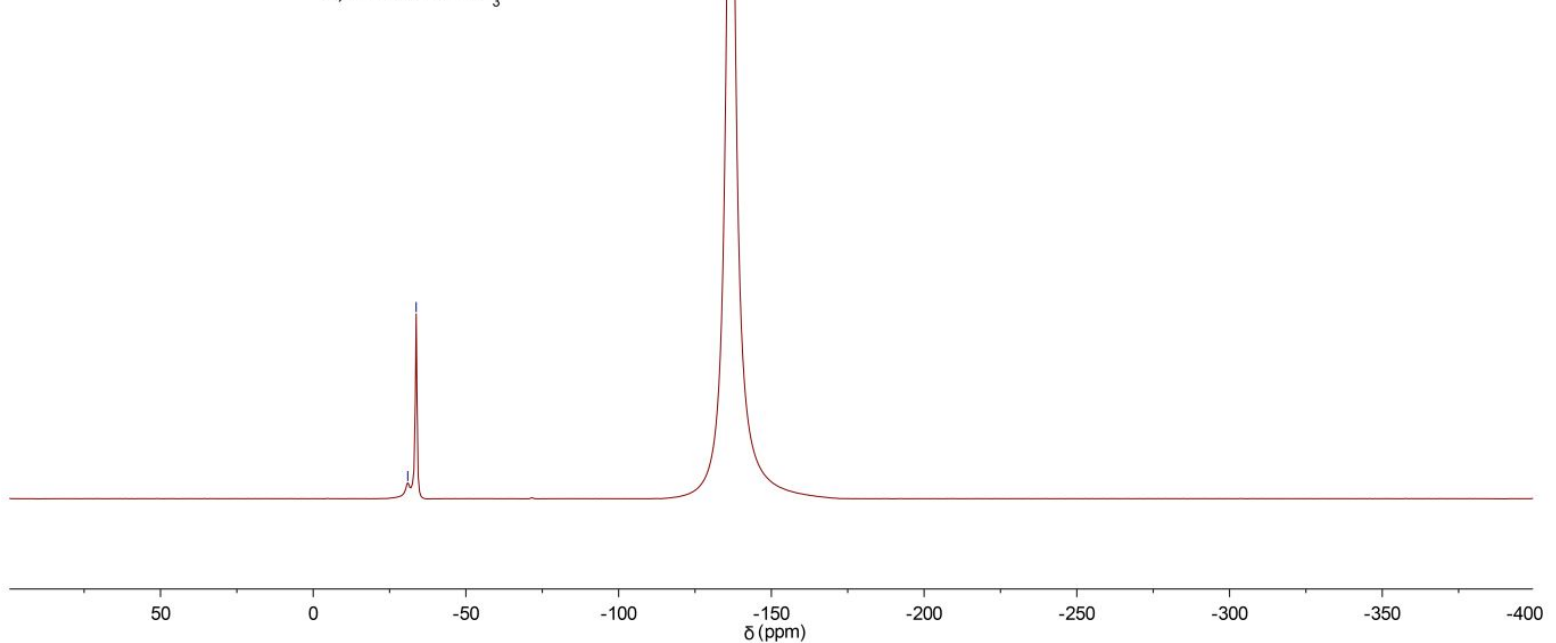




\section{Vibrational Spectroscopy}

The most characteristic vibration frequencies in the IR and Raman spectra are the carbonyl and nitro groups, which are along with $v(\mathrm{CH})$, summarized in Table S3. Thereby, the $\mathrm{CH}$-stretching modes can be found in the range $\tilde{v}=2998-2960 \mathrm{~cm}^{-1}$ and the $v(\mathrm{C}=\mathrm{O})$ stretching vibrations are located in the large range $\tilde{v}=1785-1683 \mathrm{~cm}^{-1}$. Regarding the nitro groups, vibrational analysis showed the characteristic asymmetric $\left(v_{\mathrm{as}}\left(\mathrm{NO}_{2}\right)\right)$ stretching vibrations in the range from $1618 \mathrm{~cm}^{-1}$ to $1561 \mathrm{~cm}^{-1}$. Furthermore, the symmetric $\left(v_{\mathrm{s}}\left(\mathrm{NO}_{2}\right)\right)$ stretching are found in the range $\tilde{v}=1305-1279 \mathrm{~cm}^{-1}$

Table S3. Selected IR and Raman bands of MaBTNE, nitraminodiacetic acid and NABTNE in $\mathrm{cm}^{-1}$.

\begin{tabular}{ccccccc}
\hline & \multicolumn{2}{c}{ MaBTNE (1) } & \multicolumn{2}{c}{ Nitraminodiactic acid } & \multicolumn{2}{c}{ NABTNE (2) } \\
& IR & Raman & IR & Raman & IR & Raman \\
\hline$v(\mathrm{CH})$ & 2973 & 2960 & 2967 & 2964 & $2998-2960$ & $2996-2960$ \\
$v(\mathrm{C}=\mathrm{O})$ & 1764 & 1785 & 1719 & 1683 & 1785 & 1781 \\
$v_{\text {as }}\left(\mathrm{NO}_{2}\right)$ & 1582 & 1611 & 1561 & 1568 & 1590 & 1618 \\
$v_{\mathrm{s}}\left(\mathrm{NO}_{2}\right)$ & 1297 & 1305 & 1279 & 1287 & 1290 & 1293 \\
\hline
\end{tabular}

\section{Microscope images}

Images of the crystals of MaBTNE and NABTNE were taken on a Leica S9i stereo microscope in magnifications from $500 \mu \mathrm{m}$ to $2 \mathrm{~mm}$, using a polarization filter.
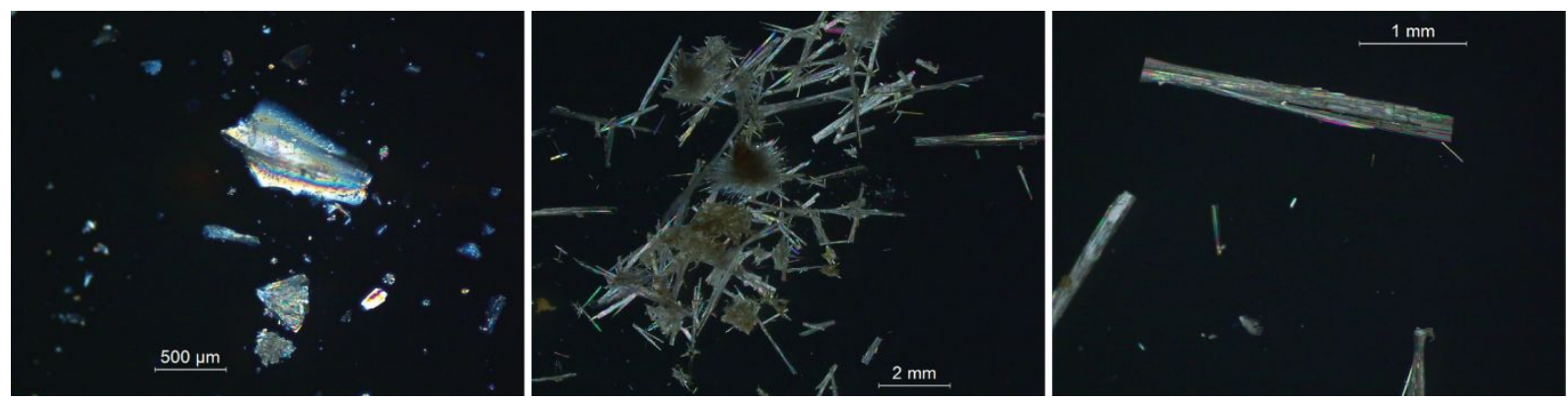

Figure S1. Microscope images of MaBTNE (left) and NABTNE (center and right). 


\section{Crystallographic Data}

\subsection{Nitraminodiacetic acid}

\begin{tabular}{|c|c|}
\hline & $\begin{array}{c}\text { Nitraminodiacetic } \\
\text { acid }\end{array}$ \\
\hline formula & $\mathrm{C}_{4} \mathrm{H}_{6} \mathrm{~N}_{2} \mathrm{O}_{6}$ \\
\hline$F W\left[\mathrm{~g} \mathrm{~mol}^{-1}\right]$ & 178.11 \\
\hline$T[\mathrm{~K}]$ & $123(2)$ \\
\hline$\lambda[\AA]$ & 0.71073 \\
\hline crystal system & orthorhombic \\
\hline space group & Pnma \\
\hline crystal size $[\mathrm{mm}]$ & $0.40 \times 0.06 \times 0.15$ \\
\hline crystal habit & colorless plate \\
\hline$a[\AA]$ & $8.8051(4)$ \\
\hline$b[\AA]$ & $14.4941(7)$ \\
\hline$c[\AA]$ & $5.2206(2)$ \\
\hline$\alpha, \beta, \gamma[\mathrm{deg}]$ & 90 \\
\hline$V\left[\AA^{3}\right]$ & $666.26(5)$ \\
\hline$Z$ & 4 \\
\hline$\rho_{\text {calc. }}\left[\mathrm{g} \mathrm{cm}^{-3}\right]$ & 1.776 \\
\hline$\mu$ & 0.170 \\
\hline$F(000)$ & 368 \\
\hline $2 \Theta$ range $[\mathrm{deg}]$ & $4.15-26.37$ \\
\hline index ranges & $\begin{array}{c}-10 \leq h \leq 11 \\
-18 \leq k \leq 18 \\
-6 \leq l \leq 6\end{array}$ \\
\hline reflections collected & 4692 \\
\hline reflections independent & 709 \\
\hline $\mathrm{R}_{\text {int }}$ & 0.0329 \\
\hline Observed reflections & 603 \\
\hline Parameters & 70 \\
\hline $\mathrm{R}_{1}$ (obs) & 0.029 \\
\hline $\mathrm{wR}_{2}$ (all data) & 0.0709 \\
\hline GooF & 1.076 \\
\hline Resd. Dens. [e $\left.\AA^{-3}\right]$ & $-0.230 ; 0.248$ \\
\hline
\end{tabular}

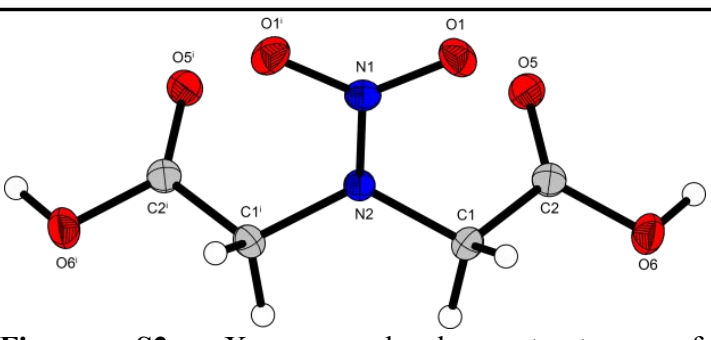

Figure S2. X-ray molecular structure of nitraminodiacetic acid. Thermal ellipsoids represent the $50 \%$ probability level.

CCDC: 2009758 


\begin{tabular}{|c|c|c|}
\hline & NABTNE & $Q^{\text {N5 }}$ \\
\hline formula & $\mathrm{C}_{8} \mathrm{H}_{8} \mathrm{~N}_{8} \mathrm{O}_{18}$ & \\
\hline$F W\left[\mathrm{~g} \mathrm{~mol}^{-1}\right]$ & 504.22 & \\
\hline$T[\mathrm{~K}]$ & $153(2)$ & \\
\hline$\lambda[\AA]$ & 0.71073 & Figure S3. X-ray molecular structure of nitramino \\
\hline crystal system & triclinic & $\begin{array}{l}\text { diacetic acid bis(trinitroethyl ester) (NABTNE). } \\
\text { Thermal ellipsoids represent the } 50 \% \text { probability level. }\end{array}$ \\
\hline space group & $P-1$ & \\
\hline crystal size $[\mathrm{mm}]$ & $0.20 \times 0.18 \times 0.14$ & CCDC: 2009759 \\
\hline crystal habit & colorless needle & \\
\hline$a[\AA]$ & $5.8695(7)$ & \\
\hline$b[\AA]$ & $17.401(2)$ & \\
\hline$c[\AA]$ & $18.116(2)$ & \\
\hline$\alpha[\mathrm{deg}]$ & $88.360(9)$ & \\
\hline$\beta[\mathrm{deg}]$ & $86.992(10)$ & \\
\hline$\gamma[\mathrm{deg}]$ & $84.906(10)$ & \\
\hline$V\left[\AA^{3}\right]$ & $1839.9(4)$ & \\
\hline$Z$ & 4 & \\
\hline$\rho_{\text {calc. }}\left[\mathrm{g} \mathrm{cm}^{-3}\right]$ & 1.82016 & \\
\hline$\mu$ & 0.182 & \\
\hline$F(000)$ & 1024 & \\
\hline $2 \Theta$ range $[\mathrm{deg}]$ & $3.38-26.37$ & \\
\hline index ranges & $\begin{aligned}-7 & \leq h \leq 6 \\
-21 & \leq k \leq 20 \\
-16 & \leq l \leq 22\end{aligned}$ & \\
\hline reflections collected & 7532 & \\
\hline reflections independent & 7532 & \\
\hline $\mathrm{R}_{\text {int }}$ & 0.0975 & \\
\hline Observed reflections & 2872 & \\
\hline Parameters & 614 & \\
\hline $\mathrm{R} 1$ (obs) & 0.0715 & \\
\hline $\mathrm{wR}_{2}$ (all data) & 01466 & \\
\hline GooF & 0.926 & \\
\hline Resd. Dens. [e $\left.\AA^{-3}\right]$ & $-0.341 ; 0.326$ & \\
\hline
\end{tabular}




\section{Plots of DSC Measurements of NABTNE (2)}

The DSC measurements were carried out on a DSC $2+$ from Mettler Toledo in heating rates of $0.5{ }^{\circ} \mathrm{C} \min ^{-1}, 1{ }^{\circ} \mathrm{C} \mathrm{min}^{-1}, 2{ }^{\circ} \mathrm{C} \mathrm{min}^{-1}, 4{ }^{\circ} \mathrm{C} \min ^{-1}$ and $8{ }^{\circ} \mathrm{C} \min ^{-1}$.

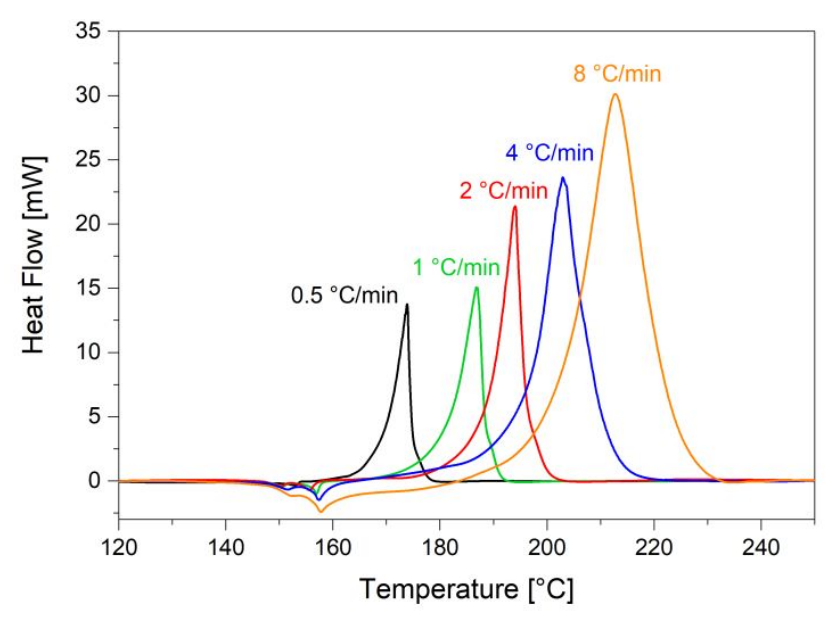

Figure S4. DSC measurements in various heating rates plotted against the resulting heat flow.

\section{Theoretical calculations}

In order to calculate the enthalpies and energies of formation, CBS4-M level calculations were used as implemented in GAUSSIAN $09 .{ }^{8-10}$ Gas phase enthalpies were transformed to solid state enthalpies by using Trouton's rule for neutral compounds. ${ }^{11}$ All ab initio calculations were carried out by using the program package Gaussian 09 and were visualized by GaussView $5.08 . .^{12}$

Based on these calculations, the detonation parameters were calculated using the EXPLO5(V6.03) program package. ${ }^{13}$ The program is based on the steady-state model of equilibrium and uses the Becker-Kistiakowski-Wilson equation of state (BKW EOS) for gaseous detonation products. EXPLO5 is designed to enable the calculation of detonation parameters at the Chapman-Jouget $(\mathrm{C}-\mathrm{J})$ point, which was found from the Hugoniot curve of the system by its first derivative. For the calculations the maximum densities at room temperature were used $(298 \mathrm{~K})$, which were calculated from the corresponding crystal densities. Therefore, the following equation and the $\alpha_{v}$ coefficient of volume expansion from the nitramine $\operatorname{HMX}\left(\alpha_{\mathrm{v}}=1.6 \cdot 10^{-4} \mathrm{~K}\right)$ was used.

$$
\rho_{298 \mathrm{~K}}=\rho_{\mathrm{T}} /\left(1+\alpha_{\mathrm{v}}(298-\mathrm{T})\right)
$$

The specific impulses $\left(I_{\mathrm{sp}}\right)$ were calculated as well at 70.0 bar chamber pressure, isobaric combustion conditions ( 1 bar) and equilibrium to throat and frozen to exit. $I_{\mathrm{sp}}$ was calculated for the neat compound, for optimized mixtures with aluminum and for a three-component 
composition with oxidizer, aluminum and $14 \%$ binder. In order to find the most suitable binder, HTPB, PBAN (6\% polybutadiene acrylic acid, $6 \%$ polybutadiene acrylonitrile and 2\% bisphenol A ether) and the more energetic binder GAP and BAMO, were taken into account. The amount of aluminum was varied from $5 \%$ to $25 \%$ and plotted against the specific impulses as shown in Graph S1 and Graph S2.

Table S4. Specific impulses of MaBTNE with varying amounts of aluminum, calculated with $14 \%$ HTPB, PBAN, GAP and BAMO as binder.

\begin{tabular}{lcccc}
\hline & HTPB & PBAN & GAP & BAMO \\
\hline$I_{\mathrm{s}} / \mathrm{s}(5.0 \% \mathrm{Al})$ & 232 & 231 & 251 & 251 \\
$I_{\mathrm{s}} / \mathrm{s}(7.5 \% \mathrm{Al})$ & 235 & 235 & 253 & 253 \\
$I_{\mathrm{s}} / \mathrm{s}(10.0 \% \mathrm{Al})$ & 240 & 240 & 255 & 255 \\
$I_{\mathrm{s}} / \mathrm{s}(12.5 \% \mathrm{Al})$ & 239 & 243 & 256 & 257 \\
$I_{\mathrm{s}} / \mathrm{s}(15.0 \% \mathrm{Al})$ & 238 & 240 & 258 & 258 \\
$I_{\mathrm{s}} / \mathrm{s}(17.5 \% \mathrm{Al})$ & 236 & 238 & 258 & 259 \\
$I_{\mathrm{s}} / \mathrm{s}(20.0 \% \mathrm{Al})$ & 234 & 235 & 256 & 256 \\
$I_{\mathrm{s}} / \mathrm{s}(22.5 \% \mathrm{Al})$ & 232 & 233 & 251 & 249 \\
$I_{\mathrm{s}} / \mathrm{s}(25.0 \% \mathrm{Al})$ & 229 & 230 & 243 & 241 \\
\hline
\end{tabular}

Graph S1. Specific impulse of MaBTNE in aluminum mixtures with different binders.

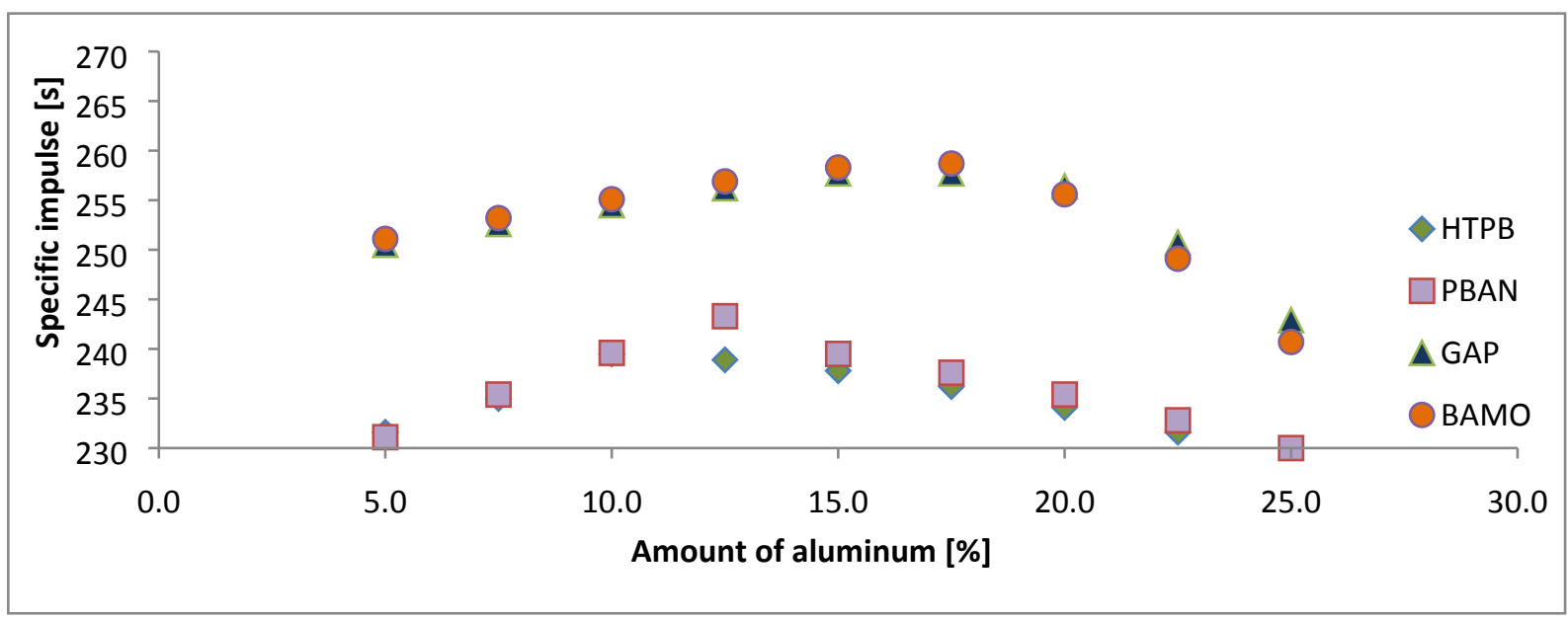


Table S5. Specific impulses of NABTNE with varying amounts of aluminum, calculated with $14 \%$ HTPB, PBAN, GAP and BAMO as binder.

\begin{tabular}{lcccc}
\hline & HTPB & PBAN & GAP & BAMO \\
\hline$I_{\mathrm{s}} / \mathrm{s}(5.0 \% \mathrm{Al})$ & 234 & 234 & 254 & 254 \\
$I_{\mathrm{s}} / \mathrm{s}(7.5 \% \mathrm{Al})$ & 238 & 238 & 256 & 256 \\
$I_{\mathrm{s}} / \mathrm{s}(10.0 \% \mathrm{Al})$ & 240 & 242 & 258 & 258 \\
$I_{\mathrm{s}} / \mathrm{s}(12.5 \% \mathrm{Al})$ & 240 & 243 & 259 & 260 \\
$I_{\mathrm{s}} / \mathrm{s}(15.0 \% \mathrm{Al})$ & 239 & 241 & 261 & 261 \\
$I_{\mathrm{s}} / \mathrm{s}(17.5 \% \mathrm{Al})$ & 238 & 239 & 261 & 261 \\
$I_{\mathrm{s}} / \mathrm{s}(20.0 \% \mathrm{Al})$ & 236 & 237 & 257 & 256 \\
$I_{\mathrm{s}} / \mathrm{s}(22.5 \% \mathrm{Al})$ & 233 & 235 & 250 & 248 \\
$I_{\mathrm{s}} / \mathrm{s}(25.0 \% \mathrm{Al})$ & 231 & 231 & 241 & 239 \\
\hline
\end{tabular}

Graph S2. Specific impulse of NABTNE in aluminum mixtures with different binders.

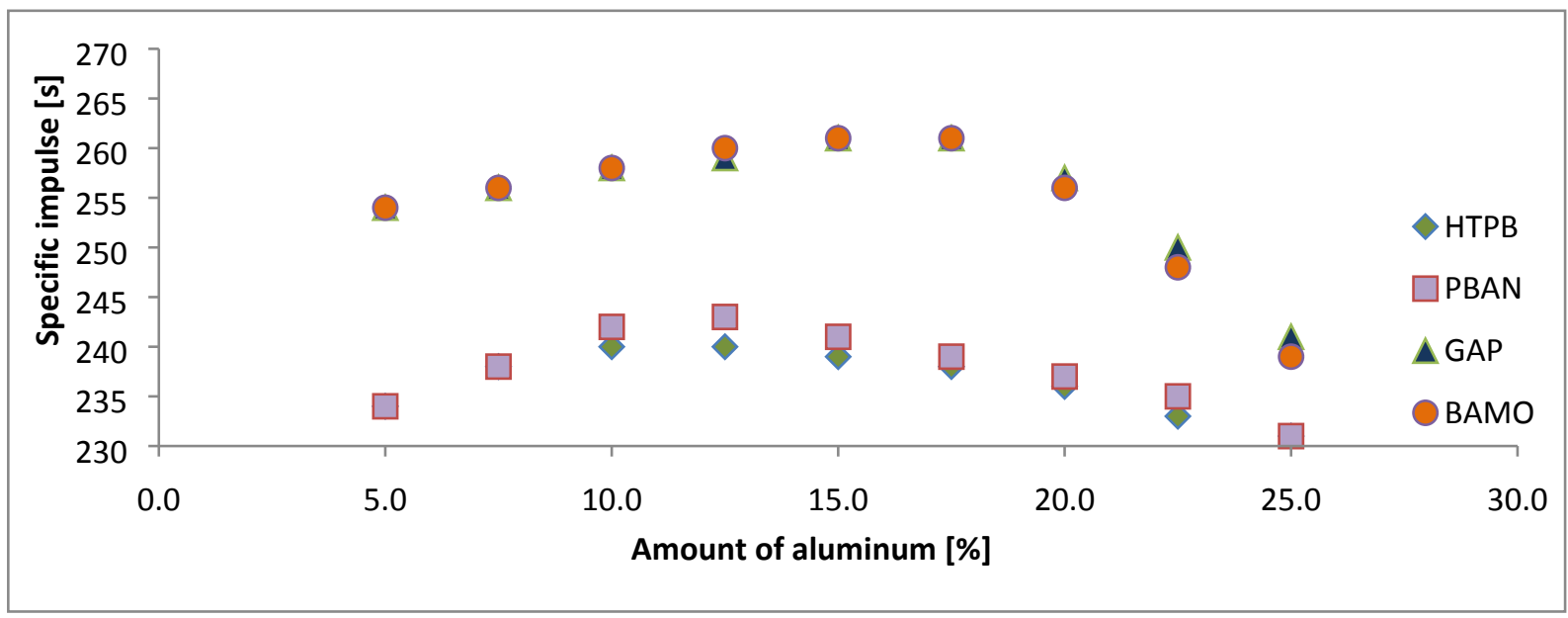




\section{References}

1. NATO. Explosives, Impact Sensitivity Tests; Standardization Agreement 4489 (STANAG 4489), Brussels, Belgium, 1999.

2. $\quad$ NATO. Explosives, Friction Sensitivity Tests; Standardization Agreement 4487 (STANAG 4487), Brussels, Belgium, 2002.

3. Altomare, A.; Cascarano, G.; Giacovazzo, C.; Guagliardi, A.; Moliterni, A. G. G.; Burla, M. C.; Polidori, G.; Camalli, M.; Spagna, R. SIR97, Univeristy of Bari (Italy), 1997.

4. Altomare, A.; Burla, M. C.; Camalli, M.; Cascarano, G. L.; Giacovazzo, C.; Guagliardi, A.; Moliterni, A. G. G.; Polidori, G.; Spagna, R., SIR97: a New Tool for Crystal Structure Determination and Refinement. J. Appl. Crystallogr. 1999, 32, 115-119.

5. Sheldrick, G. M., A Short History of SHELX. Acta Crystallogr. 2008, 64A, 112-122.

6. Sheldrick, G. M. SHELX-97, Programs for Crystal Structure Determination, University of Göttingen: University of Göttingen, Göttingen (Germany), 1997.

7. Farrugia, L. J., WinGX Suite for Small-molecule Single-crystal Crystallography. J. Appl.Crystallogr. 1999, 32, 837-838.

8. Frisch, M. J.; Trucks, G. W.; Schlegel, H. B.; Scuseria, G. E.; Robb, M. A.; Cheeseman, J. R.; G. Scalmani, V. B.; Mennucci, B.; Petersson, G. A.; Nakatsuji, H.; Caricato, M.; Li, X.; Hratchian, H. P.; Izmaylov, A. F.; Bloino, J.; Zheng, G.; Sonnenberg, J. L.; Hada, M.; Ehara, M.; Toyota, K.; Fukuda, R.; Hasegawa, J.; Ishida, M.; Nakajima, T.; Honda, Y.; Kitao, O.; Nakai, H.; Vreven, T.; J. A. Montgomery, J.; Peralta, J. E.; Ogliaro, F.; Bearpark, M.; Heyd, J. J.; Brothers, E.; Kudin, K. N.; Staroverov, V. N.; Kobayashi, R.; Normand, J.; Raghavachari, K.; Rendell, A.; Burant, J. C.; Iyengar, S. S.; Tomasi, J.; Cossi, M.; Rega, N.; Millam, J. M.; Klene, M.; Knox, J. E.; Cross, J. B.; Bakken, V.; Adamo, C.; Jaramillo, J.; Gomperts, R.; Stratmann, R. E.; Yazyev, O.; Austin, A. J.; Cammi, R.; Pomelli, C.; Ochterski, J. W.; Martin, R. L.; Morokuma, K.; Zakrzewski, V. G.; Voth, G. A.; Salvador, P.; Dannenberg, J. J.; Dapprich, S.; Daniels, A. D.; Farkas, Ö.; Foresman, J. B.; Ortiz, J. V.; Cioslowski, J.; Fox, D. J., Gaussian 09. Rev. A.02 ed., Gaussian, Inc., Wallingford CT (US), 2009.

9. Ochterski, J. W.; Petersson, G. A.; Montgomery, J. A., A Complete Basis Set Model Chemistry. V. Extensions to Six or More Heavy Atoms. J. Chem. Phys. 1996, 104, 2598-2619.

10. J. A. Montgomery; Frisch, M. J.; Ochterski, J. W.; Petersson, G. A., A Complete Basis Set Model Chemistry. VII. Use of the Minimum Population Localization Method. J. Chem. Phys. 2000, $112,6532-6542$.

11. Trouton, F., On Molecular Latent Heat. Philos. Mag. 1884, 18, 54-57.

12. Dennington II, R. D.; Keith, T. A.; Millam, J. M., GaussView. Ver. 5.08 ed., Semichem, Inc., Wallingford CT (US), 2009.

13. Sućeska, M., EXPLO5 V6.03. Zagreb (Croatia), 2015. 